\title{
Investigating eating time patterns in UK adults from The 2008-2012 National Diet and Nutrition Survey
}

\author{
R. Mansukhani and L. Palla \\ Department of Medical Statistics, The London School of Hygiene and Tropical Medicine, Keppel Street, London \\ WCIE 7HT, UK.
}

Eating later in the day is associated with obesity which in turn is associated with poorer health outcomes ${ }^{(1)}$. Currently there are few studies which have investigated eating time patterns. Leech ${ }^{(2)}$ investigated temporal eating habits in Australian adults using Latent Class Analysis(LCA). Here we use LCA to investigate the hourly eating patterns of UK adults.

Data from four daily food diaries for 2007 women and 1443 men from the 2008-2012 UK National Dietary and Nutrition Survey Rolling Programme (NDNS RP) were analysed. LCA was used to calculate the probability of whether an eating occasion had occurred for every hour of the day for individuals within each distinct class. The average number of kilojoules (kJ) was calculated for each individual for each hour of the day over the four days. We classified an eating occasion to have occurred if an average of more than $210 \mathrm{~kJ}$ had been consumed by that individual for that hour of the day. Alternative definitions of eating occasions were used but these did not change the interpretation of results ${ }^{(3)}$. We investigated whether latent class membership was associated with age, BMI, working hours, living with other people, being married and household income. The association between age and class was assessed using an $\mathrm{F}$ Test and the association between other factors and latent class was assessed using a chi ${ }^{(2)}$ test.
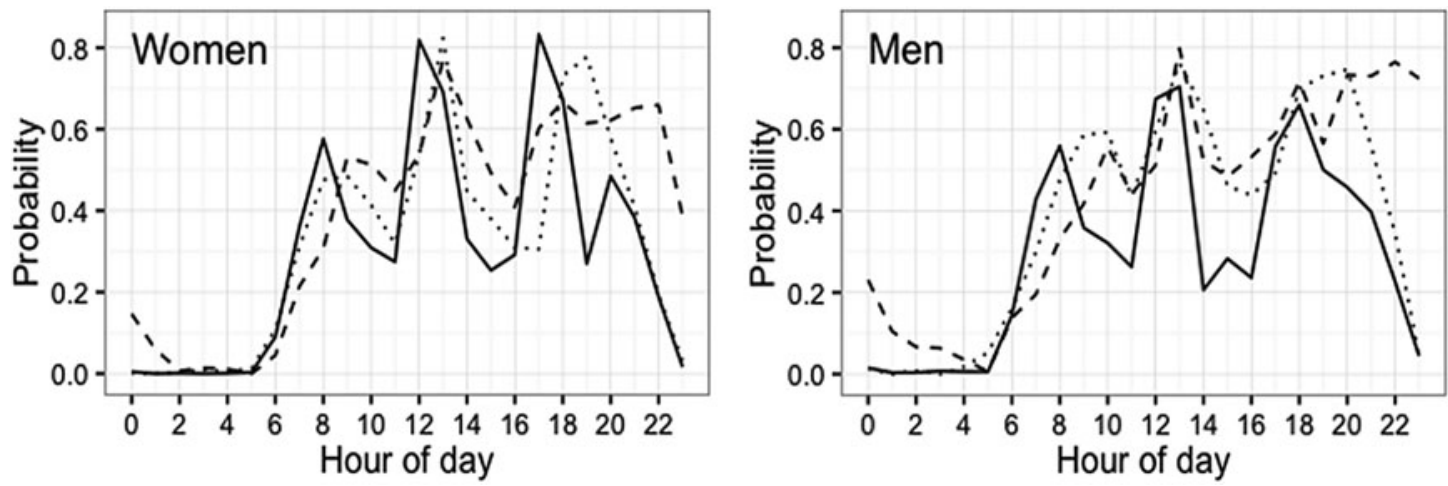

Fig. 1. The probability of an eating occasion versus hour of the day in women $(n=2007)$ and men $(n=1443)$. We identify a standard meal class (the solid line), a later meal time eating class (the dotted line) and an irregular meal time eating class (the dashed line).

We identified three patterns of eating: a standard eating class (Women 38\% Men $48 \%$ ), a later class (Women $46 \%$ Men $33 \%$ ) which were likely to eat meals slightly later and an irregular class(Women $16 \%$ Men $18 \%$ ) which were less likely to eat meals at a fixed time. We found that for both men and women ( $<<0.001$ in both cases) younger people ate later. Married as opposed to single $\operatorname{men}(p=0.002)$ ate earlier. People who are degree educated tend to eat earlier $($ men $p=0.001$ women $p<0.001)$ than their non degree educated peers. And while being in paid employment is associated with eating earlier $($ men $p<0 \cdot 001$ women $p<0 \cdot 001)$ higher household income(men $\mathrm{p}=0.050$ women $\mathrm{p}<0.001$ ) is associated with eating later. We also found that women who work more than 35 hours a week are more likely $(\mathrm{p}=0.014)$ to eat later.

Our results suggest that people fall into distinct eating classes and that later eating is associated with various socioeconomic factors. It would be interesting to next investigate how eating time patterns identified via LCA are associated to health outcomes.

1. Almoosawi et al. (2016) Proceedings of the Nutrition Society 75(4)

2. Leech et al. (2017) International Journal of Behavioural Nutrition and Physical Activity 14, 3

3. Leech et al. (2015) American Journal of Clinical Nutrition 102, 1229-37 